\title{
THERMODYNAMIC PROPERTIES OF PSEUDOSPIN-ELECTRON MODEL IN THE GAUSSIAN FLUCTUATION APPROXIMATION
}

\author{
K. V. Tabunshchyk \\ Institute for Condensed Matter Physics of the National Academy of Sciences of Ukraine, \\ 1 Svientsitskii Str., Lviv, UA-79011, Ukraine \\ Department of Physics, Queen's University, Kingston, Ontario, K' 7 L 36 Canada
}

(Received January 4, 2002; received in final form February 21, 2005)

\begin{abstract}
We investigate the thermodynamics of phase transitions in pseudospin-electron model with strong coupling, and focus on the role of fluctuation effects in the vicinity of transitions. We analyze the behaviour of grand canonical potential, and find the mean value of physical quantities as well as root-mean-square fluctuations of an internal field within the generalized random phase approximation (GRPA), the Onyszkiewicz type approximation, and self-consistent gaussian fluctuation approximation. Within all of these approximations we obtain the possibility of first order phase transitions with a jump of pseudospin mean value and reconstruction of electron spectrum. The final results are presented in the form of phase diagrams in plane temperature-longitudinal field $T_{\mathrm{c}}-h$, and we find that the fluctuation effects cause quantitative and, in some special cases, qualitative changes on the phase diagrams near a critical point. We show that taking the fluctuations into account may also lead to narrowing the temperature range where modulated (chessboard) phase can be found.
\end{abstract}

Key words: gaussian fluctuations, phase transitions, pseudospin-electron model, anharmonic systems.

PACS number(s): 63.20.Ry, 71.10.Fd, 77.80.Bh

\section{INTRODUCTION}

Properties of pseudospin-electron model (PEM) have attracted much attention in connection with the investigations of systems characterized by strong shortrange correlations between the conducting electrons, and strong coupling of the electrons with local anharmonic lattice vibrations. Such a consideration arises from the analysis of distinguishing features of copper-oxide high$T_{c}$ superconductors (HTSC). These are novel materials with a layered perovskite-type structure, and experimental data have pointed out (i) strong electron correlations related to the Hubbard type interactions in the conducting bands; (ii) strong anharmonicity in the vibrations of apical oxygen ions along the axis normal to the copper-oxide layers; and another important feature, (iii) significant correlation between the position of the apical oxygen ions and the energy of the electron states in the conducting planes. To be concrete, we can mention experimental analysis of EXAFS [1] and Raman scattering measurements $[2,3]$ on $\mathrm{YBa}_{2} \mathrm{Cu}_{3} \mathrm{O}_{7}$ crystal where two equilibrium positions of the apical oxygen ions have been observed, that can point out the presence of a local double-minimum potential well. Experimental data given by Cava et al. [4] have shown the existence of a large electron-vibration coupling between the electrons in the conducting $\mathrm{CuO}_{2}$ planes and the positions of the apical oxygen ions in the double-well potential.

In the case when two equilibrium positions of anharmonic ions really exist it is reasonable to use a pseudospin formalism; the pseudospin variable $S_{i}^{z}= \pm 1 / 2$ defines these two positions. Then, strong local coupling of the anharmonic mode with the conducting electrons can be modeled by a pseudospin-electron approach. Starting from Müller's work [5] and series of Hirsch's papers [6,7] the pseudospin-electron approximation is applied to the $\mathrm{YBaCuO}$ type systems. Recently a similar model was used by Matsushita [8,9] to investigate proton-electron interactions in molecular and crystalline systems with hydrogen bonds. In such systems the pseudospin formalism describes two equilibrium positions of a proton in the hydrogen bond. It is worth noting also that in one special case, as we shall explain below, the PEM was believed to be similar to the Falicov-Kimball models used for binary alloys.

The Hamiltonian of the PEM has the following form:

$$
H=\sum_{i} H_{i}+\sum_{i j \sigma} t_{i j} c_{i \sigma}^{+} c_{j \sigma}
$$

where the single-site part is given by

$$
\begin{aligned}
H_{i}= & -\mu\left(n_{i \uparrow}+n_{i \downarrow}\right)+U n_{i \uparrow} n_{i \downarrow} \\
& +g\left(n_{i \uparrow}+n_{i \downarrow}\right) S_{i}^{z}-h S_{i}^{z}-\Omega S_{i}^{x}
\end{aligned}
$$

Here, $c_{i \sigma}^{+}, c_{i \sigma}$ are $\sigma$-spin electron creations and annihilation operators and $n_{i \sigma}=c_{i \sigma}^{+} c_{i \sigma}$ is the electron occupation number. This Hamiltonian (1),(2) consists of the kinetic energy $\left(t_{i j}\right.$ term) together with (i) the energy of electron correlation ( $U$ term), (ii) the energy of electronpseudospin coupling ( $g$ term), (iii) the energy of anharmonic potential asymmetry that is involved in the model 
as a longitudinal filed acting on the pseudospins ( $h$ term), and (iv) the energy of tunnelling splitting involved as a transversal filed acting on the pseudospin subsystem ( $\Omega$ term). Hence, with respect to the $\mathrm{YBaCuO}$ system, the PEM describes the electronic states of $\mathrm{CuO}_{2}$ planes within the Hubbard model, and anharmonic vibrations of the apical oxygen ions within the pseudospin formalism. The term $g n_{i \sigma} S_{i}^{z}$ determines the change in the energy of an electron on the site $i$ due to the transition of the apical oxygen ion on the same site from one minimum $\left(S_{i}^{z}=+\frac{1}{2}\right)$ of the anharmonic potential to the other one $\left(S_{i}^{z}=-\frac{1}{2}\right)$. The magnitude of coupling $g$ is several electron volts due to the strong oxygen polarizability of the $\mathrm{CuO}_{2}$ planes.

Based on model $(1,2)$ a possible connection between the superconductivity and lattice instability of ferroelectric type in the HTSC systems has been discussed in the literature $[7,10]$. Investigations of the electron spectrum and electron statistics of the PEM have been performed by Stasyuk et al. [11], who have used the technique of double-time temperature-dependent Green's functions within the Hubbard-I approximation. A series of works $[12,13]$ has been carried out where pseudospin $\left\langle S^{z} S^{z}\right\rangle$, mixed $\left\langle S^{z} n\right\rangle$, and charge $\langle n n\rangle$ correlation functions were calculated within a generalized random phase approximation (GRPA) in the limit of infinite single-site electron correlations (limit of $U \rightarrow \infty$ in the Hamiltonian $(1,2)$ ). Some authors have shown a possibility of divergence of the correlation functions at certain temperature, and interpreted this result as a manifestation of dielectric instability or ferroelectric type anomaly. They have also established the tendency to forma a spatially modulated charge and pseudospin ordering. In the next work [14] within the same (GRPA) approach the instability with respect to the phase separation in the PEM with $U \rightarrow \infty$ has been also marked. We want to emphasize that in the above mentioned works authors investigated the instabilities of the correlation functions with respect to the formation of different phases, but the questions concerning the existence, transitions and thermodynamical stabilities of the phases were not considered.

The great progress in the understanding of the thermodynamic properties of the systems described by the PEM has been connected with detailed investigations of a simplified PEM $[15,16]$. The model Hamiltonian (1),(2) in the case of the zero electron correlation $U=0$ and tunnelling splitting $\Omega=0$ was originally considered in [15] within the framework of dynamical mean field approximation (DMFA), and in [16] within the self-consistent GRPA scheme. An important feature of this simplified $\mathrm{PEM}$ is that the model does not contain the direct electron-electron and the direct pseudospin-pseudospin on-site interactions, but an effective many-body pseudospin interaction via the subsystem of conducting electrons exists. Hence, from the theoretical point of view, the simplified PEM gives a good opportunity to clarify a role of the retarded nondirect interaction between the pseudospins in the appearance of phase transitions and phase separations, as well as in the modification of electron spectrum.
As we have already mentioned the PEM Hamiltonian $(1,2)$ allows one to describe the binary alloy type model. In order to prove this statement we introduce projective operators on pseudospin states $P_{i}^{ \pm}=1 / 2 \pm S_{i}^{z}$; then the Hamiltonian of the binary alloy model can be obtained by substitution $P_{i}^{+}=p_{i}, P_{i}^{-}=1-p_{i}$ where $p_{i}$ is the concentration of one component of the binary alloy, and $1-p_{i}$ is the concentration of the second one. The main difference between these two models is in the way (i) how an averaging procedure over projection operators $P^{ \pm}$is performed: thermal statistical averaging in the case of the simplified PEM, and configurational averaging for the binary alloy; and (ii) how the self-consistency is achieved: fixed value of the longitudinal field $h$ for the simplified PEM, and fixed value of the component concentration $p$ for the binary alloy.

Besides, if we remove in (1),(2) spin indices and rewrite Hamiltonian of the simplified PEM in terms of operators of the mobile $d_{i}\left(c_{i \sigma}=d_{i}\right)$ and localized $f_{i}\left(P_{i}^{+}=f_{i}^{+} f_{i}\right.$, $\left.P_{i}^{-}=1-f_{i}^{+} f_{i}\right)$ electrons, we shall get the Hamiltonian of the Falicov-Kimball (FK) model

$$
\begin{aligned}
H_{F K}= & \sum_{i j} t_{i j} d_{i}^{+} d_{j}+U \sum_{i} d_{i}^{+} d_{i} f_{i}^{+} f_{i} \\
& -\mu_{f} \sum_{i} f_{i}^{+} f_{i}-\mu_{d} \sum_{i} d_{i}^{+} d_{i}
\end{aligned}
$$

where the field $h / 2$ in the PEM plays the role of chemical potential for the localized $f$-electrons in the FK model $\left(\mu_{f}\right.$ term), the parameter $\mu+g / 2$ in the PEM plays the role of a chemical potential for the mobile $d$-electrons in the FK model ( $\mu_{d}$ term), and the coupling constant $g$ in the PEM describes strong local interaction between the mobile and localized particles in the FK model ( $U$ term).

Detailed investigations of the simplified PEM within the self-consistent GRPA approach have been made in the series of works [16-18]. This novel approach was originally proposed in work [16] for the simplified PEM and then was generalized for the Hubbard type models with an infinite on-site repulsion [19]. The self-consistent GRPA allows us to calculate the grand canonical potential, pseudospin mean value, electron concentration together with the correlation functions. We have shown that this approximation does not violate the electron-hole symmetry in the model, and all obtained phase diagrams completely satisfy the internal Hamiltonian symmetry of the simplified PEM: $\mu \rightarrow-\mu, h \rightarrow 2 g-h, n \rightarrow 2-n$, $S^{z} \rightarrow-S^{z}$.

In our works [16-18] we identified two different regimes in which the system can exist:

1) the $\mu=$ const regime; it is supposed that electrons of other structural elements, which are not included explicitly into the model, play the role of thermostat ensuring the fixed value of the chemical potential $\mu$ (despite the possible changes of temperature and parameters of the model). In this case a statistical ensemble of the system is described by the grand canonical one. Our investigations have shown that in the $\mu=$ const regime the first order 
phase transitions between the different uniform phases take place when the chemical potential is located within the lower or upper electron subband of spectrum. Obtained within the self-consistent GRPA scheme phase diagrams quite sufficiently coincide with corresponding ones calculated in the DMFA [15]. Further, the comparison of the grand canonical potential for the uniform and modulated chessboard phases has led to the conclusion that at low temperature the modulated phase is thermodynamically stable when the chemical potential is placed in the gap of electron spectrum or in the lower (upper) subband close to the gap. In the latter case the interband transitions in the effective many-body interaction prevail over intraband ones. We have shown possibilities of the second and first order phase transitions between the uniform and modulation phases. Our investigations have disclosed that the electronic band structure is determined by the pseudospin mean value, as expected, and a jump of this mean value at the first order transition point is accompanied by the electron spectrum reconstruction.

Note that our results extended the existing ones for the FK model [20], where authors have made an assumption that the transitions to the chessboard phase are always continuous. We have also analyzed the temperature behaviour of the correlation functions, and shown the impossibility of an incommensurate phase appearing in the strong-coupling limit $(g \gg t)$ of the simplified PEM. The same conclusion has been obtained in work [21] for the FK model an other thermodynamic regime.

2 ) the $n=$ const regime; this situation is more customary at the consideration of electron systems, and means that now the chemical potential is a function of $T, h$, electron concentration, etc. The analysis of the chemical potential and free energy behaviours versus the electron concentration has shown the existence of the phase separation in the electron and pseudospin subsystems. We have revealed two possibilities (i) phase separation into the states with the uniform (but different) pseudospin mean values and electron concentrations; and (ii) separation into the uniform phase and the modulated chessboard phase. The former one is caused by the leading role of the intraband transitions, while the latter one, i.e. the appearance of the phase with the chessboard modulation, has been explained as a result of the interband transitions (effects associated with the many-body interaction). We have also investigated the behavior of the pseudospin subsystem in the separated phases, and changes in the electron spectrum and in the density of states of the electron bands. At one time, the competition between the phase separation and charge-densitywave ordering has been obtained for the FK model in the strong coupling case within the DMFA [20]. But the comprehensive analysis of phase stability was not made. Therefore, in the work [20] (just as it has been done in other works [22-24]) phase diagrams for the FK model within the DMFA have been obtained by using the Maxwell construction, or based on the analysis of correlation functions divergences. Such investigations are not enough to build the correct phase diagrams in the case when phase separation to the regions with different mod- ulated phases can exist; the investigation of the free energy are required (see our paper [18]).

In the present paper we continue our consideration of the simplified PEM, and calculate the thermodynamic and correlation functions by taking into account the fluctuations of the effective self-consistent field [25]. We emphasize that our study of the simplified PEM is not expected to explain some of the observed properties of a real system; in fact, this work stands only as a theoretical study of the role of the fluctuation effects on the thermodynamics of phase transitions in the model. The only regime of the fixed chemical potential value ( $\mu=$ const) is considered (actually in this regime the possibility of phase transitions has been established). We consider the case of the strong local interaction $(g \gg t)$, and discuss the applicability of the schemes previously used to the model. For this purpose the results of the investigations within the self-consistent GRPA [16] and the DMFA [15] are presented together with the recent examinations (i. e. within the scheme that allows one to take the fluctuation effects into account).

Our paper is organized as follows. In order to put the paper in a self-contained form a short review of the selfconsistent GRPA is presented in Section II, and several new results calculated within the GRPA scheme are presented and compared with the corresponding ones obtained within the DMFA [15]. In Section III, we present the self-consistent gaussian fluctuation approximation for the PEM, and give the simplified version of this approximation (analogous to the approximation proposed by the Onyszkiewicz $[26,27]$ for spin models). Then, in Section IV we present an examination of numerical results calculated within the gaussian fluctuation method and the Onyszkiewicz method, including a comparison with the GRPA results. Finally, in Section V we summarize our paper and give conclusions.

\section{SELF-CONSISTENT GRPA METHOD}

For the first time GRPA method was formulated by Izyumov et al. [28], and authors have applied this method to the Hubbard and the $t-J$ models. The GRPA allows one to investigate the magnetic susceptibility of the system, and takes into account the same topological class of diagrams (so-called loop diagrams) as the traditional random phase approximation (RPA) does. The main difference between the GRPA and the RPA is a way of choosing the basic states: splitt (HubbardI) band states in the GRPA and pure band states in the RPA. The question how to calculate the thermodynamic quantities within the GRPA has been open until recent works $[16,19]$. In this section we briefly show how to construct the self-consistent GRPA for the simplified PEM. An important feature of this method is that it can be used to obtain both thermodynamic and correlation functions [16].

We perform our calculations in the strong coupling case $(g \gg t)$ using the single-site states as the basic ones. The formalism of electron annihilation (creation) 
operators

$$
\begin{array}{ll}
a_{i \sigma}=c_{i \sigma} P_{i}^{+}, & \tilde{a}_{i \sigma}=c_{i \sigma} P_{i}^{-}, \\
a_{i \sigma}^{+}=c_{i \sigma}^{+} P_{i}^{+}, & \tilde{a}_{i \sigma}^{+}=c_{i \sigma}^{+} P_{i}^{-}, \quad\left(P_{i}^{ \pm}=\frac{1}{2} \pm S_{i}^{z}\right)
\end{array}
$$

acting on a site with certain pseudospin orientation is introduced [16]. Within the proposed representation (4), the initial Hamiltonian of the simplified PEM (i.e. Hamiltonian $(1,2)$ in the limit $U=0, \Omega=0$ ) has the form

$$
\begin{aligned}
& H=H_{0}+H_{\mathrm{int}}=\sum_{i \sigma}\left\{\varepsilon_{1} n_{i \sigma}+\varepsilon_{2} \tilde{n}_{i \sigma}-\frac{h}{2} S_{i}^{z}\right\} \\
& +\sum_{i j \sigma} t_{i j}\left(a_{i \sigma}^{+} a_{j \sigma}+a_{i \sigma}^{+} \tilde{a}_{j \sigma}+\tilde{a}_{i \sigma}^{+} a_{j \sigma}+\tilde{a}_{i \sigma}^{+} \tilde{a}_{j \sigma}\right) .
\end{aligned}
$$

Here

$$
\begin{aligned}
& n_{i \sigma}=a_{i \sigma}^{+} a_{i \sigma}=c_{i \sigma}^{+} c_{i \sigma} P_{i}^{+} \\
& \tilde{n}_{i \sigma}=\tilde{a}_{i \sigma}^{+} \tilde{a}_{i \sigma}=c_{i \sigma}^{+} c_{i \sigma} P_{i}^{-}
\end{aligned}
$$

are the electron occupation operators acting on a site with a certain pseudospin orientation, and

$$
\varepsilon_{1,2}=-\mu \pm g / 2
$$

are the energies of the single-site states.

The perturbation expansion in the electron transfer $H_{\text {int }}(5)$ leads to the infinite series of terms containing the averages of the $T$-products of the $a_{i \sigma}, \tilde{a}_{i \sigma}, a_{i \sigma}^{+}, \tilde{a}_{i \sigma}^{+}$ operators. The evaluation of such averages is made using the appropriate Wick's theorem [16] formulated in the spirit of the Wick's theorem for the Hubbard operators [29]. This theorem gives an algorithm reducing the average of the product of $n$ creation (annihilation) operators to the sum of averages of $n-2$ operators. The result is expressed in terms of the products of nonperturbed Green's functions and averages of the projection operators $P_{i}^{ \pm}$, the latter are calculated by means of the semi-invariants expansion.

Nonperturbated electron Green's function is

$$
g\left(\omega_{n}\right)=\left\langle g_{i}\left(\omega_{n}\right)\right\rangle
$$

$$
g_{i}\left(\omega_{n}\right)=\frac{P_{i}^{+}}{i \omega_{n}-\varepsilon_{1}}+\frac{P_{i}^{-}}{\mathrm{i} \omega_{n}-\varepsilon_{2}}
$$

where $\omega_{n}=\frac{2 n+1}{\beta} \pi$ for $n \in \mathbb{Z}$ are the Fermi Matsubara frequencies. Then, in the uniform case $\left\langle S_{i}^{z}\right\rangle=\left\langle S^{z}\right\rangle$, we can write down a single-electron Green's function within the Hubbard-I type approximation as

$$
\Longrightarrow=G_{\boldsymbol{k}}\left(\omega_{n}\right)=\left(g^{-1}\left(\omega_{n}\right)-t_{k}\right)^{-1} \text {. }
$$

In [16] we have shown that the Hubbard-I type approximation does not violate the particle-hole symmetry of the simplified PEM, i.e. our results are invariant with respect to the transformation $\mu \rightarrow-\mu, h \rightarrow 2 g-h$, $n \rightarrow 2-n, S^{z} \rightarrow-S^{z}$

Poles of the Green's function $G_{\boldsymbol{k}}\left(\omega_{n}\right)$ determine the electron spectrum

$$
\varepsilon_{\mathrm{I}, \mathrm{II}}\left(t_{\boldsymbol{k}}\right)=-\mu+\frac{t_{\boldsymbol{k}}}{2} \pm \frac{1}{2} \sqrt{g^{2}+4 t_{\boldsymbol{k}}\left\langle S^{z}\right\rangle g+t_{\boldsymbol{k}}^{2}} .
$$

Two branches $\varepsilon_{\mathrm{I}}\left(t_{\boldsymbol{k}}\right)$ and $\varepsilon_{\mathrm{II}}\left(t_{\boldsymbol{k}}\right)$ of the spectrum form two electron subbands always separated by a gap at $g \neq 0$. Reconstruction of the electron spectrum (10) takes place with the change of the pseudospin mean value $\left\langle S^{z}\right\rangle$.

In the self-consistent GRPA scheme the diagrammatic series for the pseudospin mean value can be presented in the following form [16]

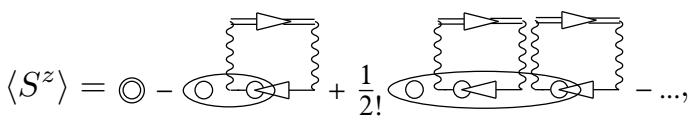

where the corresponding diagrammatic notations are used: $\bigcirc-S^{z}, \smile--g_{i}\left(\omega_{n}\right)$, wavy line is the Fourier transform of the hopping integral $t_{\boldsymbol{k}}$. The semi-invariants are represented by ovals and contain $\delta$-symbols on site indices.

In Eq. (11) we take the renormalization of the basic semi-invariants in the spirit of the mean field type approach, i. e. we insert independent loop fragments in the all ovals. It should be noted that within the selfconsistent GRPA scheme in the sequence of diagrams the connections between any two loops by more than one semi-invariant are omitted. The analytical expression for the loop is the following

$$
\sum_{G}\left\{=\frac{2}{N} \sum_{n, \boldsymbol{k}} \frac{t_{\boldsymbol{k}}^{2}}{g^{-1}\left(\omega_{n}\right)-t_{\boldsymbol{k}}}\left(\frac{P_{i}^{+}}{i \omega_{n}-\varepsilon_{1}}+\frac{P_{i}^{-}}{i \omega_{n}-\varepsilon_{2}}\right)=\beta\left(\alpha_{1} P_{i}^{+}+\alpha_{2} P_{i}^{-}\right),\right.
$$

where expressions for symbols $\alpha_{1,2}$ are defined according to the written formula (12).

It is easy to show, that summation of the diagrammatic series (11) for the pseudospin mean value is equivalent to 
averaging with the mean-field type Hamiltonian

$$
H_{\mathrm{MF}}=\sum_{i}\left\{\varepsilon_{1}\left(n_{i \uparrow}+n_{i \downarrow}\right)+\varepsilon_{2}\left(\tilde{n}_{i \uparrow}+\tilde{n}_{i \downarrow}\right)-y S_{i}^{z}\right\},
$$

where expression

$$
\begin{aligned}
y & =h+\alpha_{2}-\alpha_{1}=h+\frac{2}{N \beta} \sum_{n, \boldsymbol{k}} \frac{t_{\boldsymbol{k}}^{2}}{g^{-1}\left(\omega_{n}\right)-t_{\boldsymbol{k}}}\left(\frac{1}{i \omega_{n}-\varepsilon_{1}}+\frac{1}{i \omega_{n}-\varepsilon_{2}}\right) \\
& =h+\frac{2}{N} \sum_{\boldsymbol{k}} \frac{g t_{\boldsymbol{k}}}{\varepsilon_{\mathrm{I}}\left(t_{\boldsymbol{k}}\right)-\varepsilon_{\mathrm{II}}\left(t_{\boldsymbol{k}}\right)}\left[n_{\mathrm{F}}\left(\varepsilon_{\mathrm{I}}\left(t_{\boldsymbol{k}}\right)\right)-n_{\mathrm{F}}\left(\varepsilon_{\mathrm{II}}\left(t_{\boldsymbol{k}}\right)\right)\right], \quad n_{\mathrm{F}}(\varepsilon)=\frac{1}{1+\mathrm{e}^{\beta \varepsilon}}
\end{aligned}
$$

determines an internal effective self-consistent field formed by retarded many-body interaction between pseudospins via conducting electrons.

Finally, the equation for the pseudospin mean value in the uniform case is found to be

$$
\left\langle S_{l}^{z}\right\rangle=\left\langle S^{z}\right\rangle_{\mathrm{MF}}=\frac{\operatorname{Tr}\left(S_{l}^{z} \mathrm{e}^{-\beta \mathrm{H}_{\mathrm{MF}}}\right)}{\operatorname{Tr}\left(\mathrm{e}^{-\beta \mathrm{H}_{\mathrm{MF}}}\right)}=b(y),
$$

where

$$
b(y)=\frac{1}{2} \tanh \left\{\frac{\beta}{2} y+\ln \frac{1+\mathrm{e}^{-\beta \varepsilon_{1}}}{1+\mathrm{e}^{-\beta \varepsilon_{2}}}\right\} .
$$

An analytical expression for mean value of particle number operator can be presented in the following form [16]

$$
\begin{array}{r}
\langle n\rangle=\frac{2}{N} \sum_{\boldsymbol{k}}\left[n_{\mathrm{F}}\left(\varepsilon_{\mathrm{I}}\left(t_{\boldsymbol{k}}\right)\right)+n_{\mathrm{F}}\left(\varepsilon_{\mathrm{II}}\left(t_{\boldsymbol{k}}\right)\right)\right] \\
-\left[1+2\left\langle S^{z}\right\rangle\right] n_{\mathrm{F}}\left(\varepsilon_{2}\right)-\left[1-2\left\langle S^{z}\right\rangle\right] n_{\mathrm{F}}\left(\varepsilon_{1}\right) .
\end{array}
$$

A diagrammatic equation on the pseudospin correlator $\left\langle S^{z} S^{z}\right\rangle_{\boldsymbol{q}}$ within the self-consistent GRPA can be written as

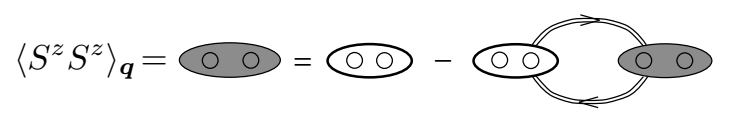

Eq. (18) differs from the corresponding one for the Ising model in the RPA by the replacement of a direct exchange interaction by the electron loop which describe the many-body retarded interaction between pseudospins via conducting electrons

$$
\begin{aligned}
\Pi_{\boldsymbol{q}}= & \frac{2}{N} \sum_{n, \boldsymbol{k}} \frac{t_{\boldsymbol{k}}}{1-t_{\boldsymbol{k}} g\left(\omega_{n}\right)} \frac{t_{\boldsymbol{k}+\boldsymbol{q}}}{1-t_{\boldsymbol{k}+\boldsymbol{q}} g\left(\omega_{n}\right)} \\
& \quad \times\left(\frac{1}{\left(i \omega_{n}-\varepsilon\right)}-\frac{1}{\left(i \omega_{n}-\tilde{\varepsilon}\right)}\right)^{2} \\
= & \frac{2}{N} \sum_{n, \boldsymbol{k}} \Lambda_{n}^{2} \tilde{t}_{n}(\boldsymbol{k}) \tilde{t}_{n}(\boldsymbol{k}+\boldsymbol{q}),
\end{aligned}
$$

where

$$
\begin{aligned}
\Lambda_{n} & =\frac{g}{\left(i \omega_{n}+\mu\right)^{2}-g^{2} / 4}, \\
\tilde{t}_{n}(\boldsymbol{k}) & =\frac{t_{\boldsymbol{k}}}{\left(1-g\left(\omega_{n}\right) t_{\boldsymbol{k}}\right)} .
\end{aligned}
$$

The first term in Eq. (18) is the zero-order correlator renormalized due to the inclusion of the mean-field type contributions ('single-tail' parts) in all basic semiinvariants. Such contributions coming from the effective pseudospin interaction and, therefore, are calculated with the help of the Hamiltonian $H_{\mathrm{MF}}$ (Eq. (13))

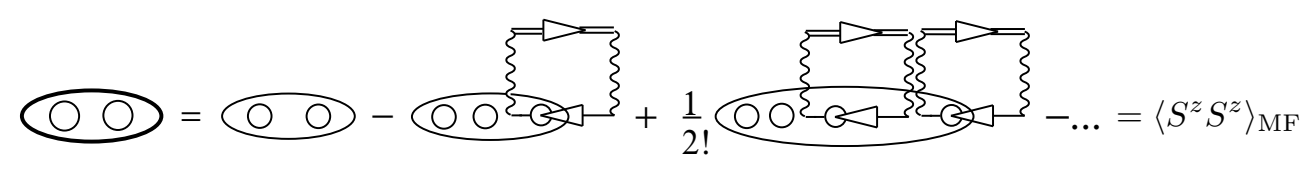




$$
=\frac{\operatorname{Tr}\left(S^{z} S^{z} \mathrm{e}^{-\beta \mathrm{H}_{\mathrm{MF}}}\right)}{\operatorname{Tr}\left(\mathrm{e}^{-\beta \mathrm{H}_{\mathrm{MF}}}\right)}=\frac{1}{4}-\left\langle S^{z}\right\rangle_{\mathrm{MF}}^{2}
$$

In the analytical form, solution of Eq. (18) turns out to be

$$
\left\langle S^{z} S^{z}\right\rangle_{\boldsymbol{q}}=\frac{1 / 4-\left\langle S^{z}\right\rangle^{2}}{1+\frac{2}{N} \sum_{n, \boldsymbol{k}} \Lambda_{n}^{2} \tilde{t}_{n}(\boldsymbol{k}) \tilde{t}_{n}(\boldsymbol{k}+\boldsymbol{q})\left(1 / 4-\left\langle S^{z}\right\rangle^{2}\right)},
$$

and we find that the pseudospin correlator is different from zero only in a static case. This comes from the fact that the pseudospin operator commutes with the initial Hamiltonian.

Within the same approach, the grand canonical potential can be written in the following analytical form [16]

$$
\begin{aligned}
& \Omega_{\mathrm{GRPA}}=-\frac{2}{N \beta} \sum_{n, \boldsymbol{k}} \ln \left(1-t_{\boldsymbol{k}} g\left(\omega_{n}\right)\right) \\
& -\frac{2}{N \beta} \sum_{n, \boldsymbol{k}} \frac{g\left(\omega_{n}\right) t_{\boldsymbol{k}}^{2}}{g^{-1}\left(\omega_{n}\right)-t_{\boldsymbol{k}}}-\frac{1}{\beta} \ln \operatorname{Tr}\left(\mathrm{e}^{-\beta H_{\mathrm{MF}}}\right) .
\end{aligned}
$$

We can verify that all the above presented physical quantities can be derived from the expression on the grand canonical potential

$$
\begin{aligned}
& \frac{d \Omega}{d(-\mu)}=\langle n\rangle, \quad \frac{d \Omega}{d(-h)}=\left\langle S^{z}\right\rangle, \\
& \frac{d\left\langle S^{z}\right\rangle}{d(\beta h)}=\left\langle S^{z} S^{z}\right\rangle_{\boldsymbol{q}=0} .
\end{aligned}
$$

This demonstrates the thermodynamic consistence of the proposed GRPA scheme.

In the $\mu=$ const regime we use the grand canonical ensemble, and, thus, the equilibrium conditions are determined from the minimum of the grand canonical potential $\Omega$. In our works [16-18] we have performed detailed numerical examination of the grand canonical potential, the pseudospin mean value, and the pair pseudospin correlation function. We have found that in the case when the chemical potential $\mu$ is placed within the gap of the electron spectrum, the uniform phase becomes unstable with respect to fluctuations with the wave vector $\boldsymbol{q}=(\pi, \pi)$ [18]. As a result, the possibility of second order phase transitions between the uniform and chessboard phase exists (at the change of temperature or field). Next, we have established that the first order phase transitions between different uniform phases (bistability effect) at the change of temperature $T$ or field $h$ can be realized in the case when the chemical potential $\mu$ is placed within the electron subband (lower or upper).

In the present paper time we study the simplified PEM in the latter case, i. e. in the case when the chemical potential is fixed and placed within the electron subband. Thus, we analyze the thermodynamics of the first order phase transitions, which are characterized by the jump of the pseudospin mean value, see figure 1a. As we mentioned above, the electron band structure is determined by the pseudospin mean value. Therefore, the jump like change of the pseudospin mean value in the first order transition point is accompanied by a rapid reconstruction of the electron spectrum, figure $1 b$ (see $[16,17]$ for details).

a)

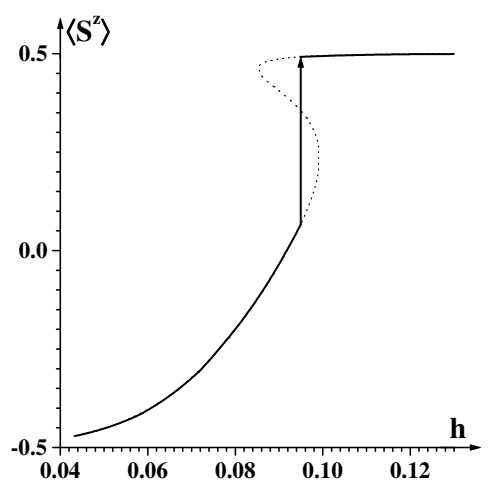

b)

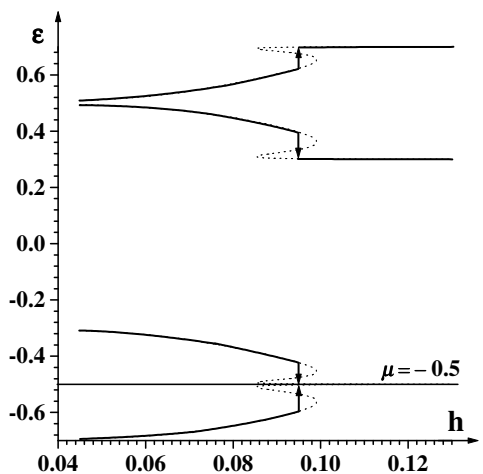

Fig. 1. Field dependences of a) pseudospin mean value, and $\mathrm{b}$ ) electron bands boundaries; in the $\mu=$ const regime when the chemical potential is placed in the lower subband of the electron spectrum. (The self-consistent GRPA scheme; $\left.g=1, t_{k=0}=0.2, \mu=-0.5, T=0.0132\right)$.

It is worth noting that the simplified PEM was considered in work [15] within the DMFA scheme. In figures $2 \mathrm{a}, \mathrm{b}$ we show the phase diagrams of the first order 
transitions within the self-consistent GRPA and DMFA methods. In figures $1 \mathrm{a}, \mathrm{b}$ and $2 \mathrm{a}, \mathrm{b}$ we present the case when the chemical potential is placed in the center of the lower electron subband $\mu=-0.5$. If the chemical potential is placed in the upper subband, the results transform according to the symmetry of the model

$$
\begin{array}{ll}
\mu \rightarrow-\mu, & h \rightarrow 2 g-h, \\
n \rightarrow 2-n, & S^{z} \rightarrow-S^{z}
\end{array}
$$

a)

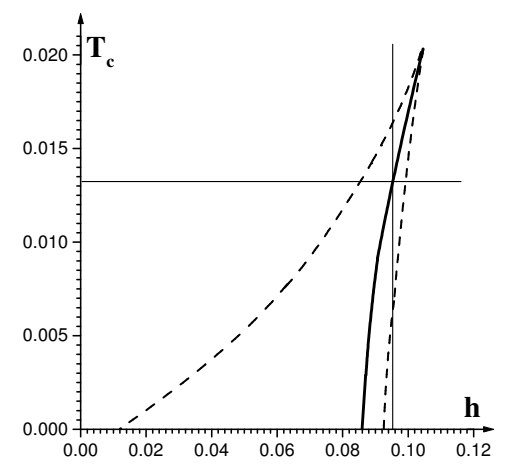

b)

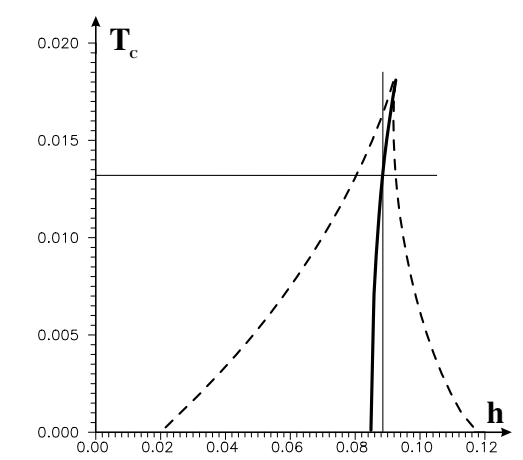

Fig. 2. $T_{\mathrm{c}}-h$ phase diagrams a) within the self-consistent GRPA, b) within the DMFA [15]. Solid and dashed lines indicate the first order phase transition line and boundaries of the phase stability (spinodal lines), respectively $(g=1$, $\left.t_{k=0}=0.2, \mu=-0.5\right)$.

One can see a quite sufficient coincidence of shapes of the first order phase transition curves (thick solid line in figures $2 \mathrm{a}, \mathrm{b})$. But, unlike the phase diagram in figure $2 \mathrm{~b}$ obtained within the DMFA, the boundaries of the phase stability region calculated in the self-consistent GRPA scheme possess the same slope. Thus, we can find a region where the vertical line twice crosses the boundary of the phase stability region (figure 2a, the GRPA calculation). The analysis of the $\left\langle S^{z} S^{z}\right\rangle$ correlator behaviour in this region (i.e.the field $h=0.0875$, the chemical potential $\mu=-0.5)$ has shown that with the decrease of temperature a high temperature phase becomes unstable with respect to fluctuations with the wave vector $\boldsymbol{q}=0$ at the lower crossing point of the vertical line and boundary of the phase stability region (dashed line in figure 2a). Similar results were obtained previously in works $[12,13]$ for temperature behaviour of the correlation functions in the case of infinite single-site electron correlation $U \rightarrow \infty$ within the GRPA method.

At the end of this paragraph let us give a short discussion of another thermodynamical regime which is more customary at the consideration of the electron system. In the regime of the fixed value of the electron concentration $n=$ const we use the canonical ensemble, and the equilibrium conditions are determined by the minimum of free energy (see our works $[16,18]$ or paper [15] for more details). In this regime the first order phase transition with a jump of the order parameter and electron concentration transforms into a phase separation into the regions with the different electron concentrations and pseudospin mean values. For the first time the instability with respect to the phase separation in the PEM was marked in work [14], where authors have obtained such an instability within the GRPA scheme in the limit of the infinite single-site electron correlation.

\section{A SELF-CONSISTENT GAUSSIAN FLUCTUATION APPROXIMATION}

The above considered approximation takes into account only the contributions of the mean field type. In this paragraph of the paper we present a self-consistent gaussian fluctuation approximation. This approximation allows one to calculate the thermodynamic and correlation functions, and takes into account the fluctuations of the self-consistent mean field [25]. As we shall show below, the self-consistent gaussian fluctuation approximation can be easily reduced to a more suitable one for numerical calculations. The Onyszkiewicz method that takes into account a restricted, in a certain way, class of diagrams. Such a method has been proposed by the Onyszkiewicz for spin models with a direct interaction [26,27], and yields a much better description of critical properties of the spin models in the whole range of temperature in comparison with other analytical schemes.

In constructing a higher order approximation that takes into account the fluctuation effects of the mean field, we use the self-consistent GRPA scheme as the zero-order approximation. This means that all 'singletail' parts of the diagrams (11),(21) have been already summed up, i. e. all semi-invariants are calculated using the distribution with the effective Hamiltonian $H_{\mathrm{MF}}$ (13). Now all these semi-invariants are represented graphically by thick ovals, and they contain the $\delta$ symbols on site index

$$
\begin{aligned}
& \text { ( }=\left\langle S^{z}\right\rangle_{\mathrm{MF}}=b(y), \\
& \text { O }=\left\langle S^{z} S^{z}\right\rangle_{\mathrm{MF}}^{\mathrm{c}}=\frac{\partial b(y)}{\partial \beta y}, \\
& \text { OO }=\left\langle S^{z} S^{z} S^{z}\right\rangle_{\mathrm{MF}}^{\mathrm{c}}=\frac{\partial^{2} b(y)}{\partial(\beta y)^{2}} .
\end{aligned}
$$


As an approximation that goes beyond the selfconsistent GRPA scheme we use (similarly to $[30,31]$ ) the method of summation of the so-called 'double-tail' diagrams. A corresponding diagrammatic series for the pseudospin mean value can be written as

$$
\left\langle S^{z}\right\rangle=
$$

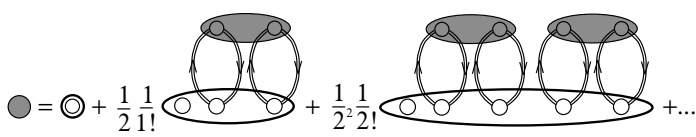

The diagrammatic equation for the pseudospin correlator $\left\langle S^{z} S^{z}\right\rangle_{\boldsymbol{q}}$ within the adopted here approximation is similar to the corresponding one within the self-consistent GRPA scheme (18)

$$
\left\langle S^{z} S^{z}\right\rangle_{\boldsymbol{q}}=
$$

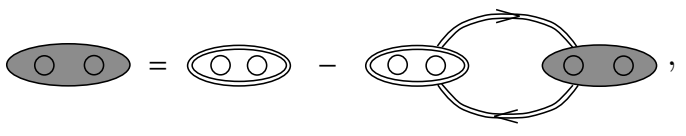

but now all semi-invariants in this equation are renormalized due to the insertion of the 'double-tail' parts

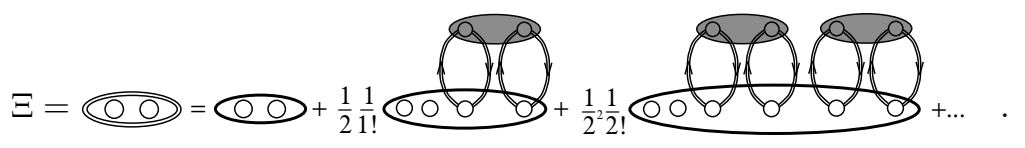

The contribution that corresponds to the 'double-tail' fragment of the diagrams can be written in the following analytical form

$$
X=\bigcap^{q}=\frac{2^{2}}{N^{3}} \sum_{n, n^{\prime}} \sum_{\boldsymbol{k}, \boldsymbol{k}^{\prime}} \sum_{\boldsymbol{q}} \Lambda_{n}^{2} \tilde{t}_{n}(\boldsymbol{k}) \tilde{t}_{n}(\boldsymbol{k}-\boldsymbol{q})\left\langle S^{z} S^{z}\right\rangle_{\boldsymbol{q}} \Lambda_{n^{\prime}}^{2} \tilde{t}_{n^{\prime}}\left(\boldsymbol{k}^{\prime}\right) \tilde{t}_{n^{\prime}}\left(\boldsymbol{k}^{\prime}+\boldsymbol{q}\right)
$$

where we used the notation from Eq. (19). The equation on the pseudospin correlation function (28) has the following solution

$$
\left\langle S^{z} S^{z}\right\rangle_{\boldsymbol{q}}=\frac{\Xi}{1+\frac{2}{N} \sum_{n, \boldsymbol{k}} \Lambda_{n}^{2} \tilde{t}_{n}(\boldsymbol{k}) \tilde{t}_{n}(\boldsymbol{k}+\boldsymbol{q}) \Xi}
$$

Since the pseudospin correlator (31) is a frequency independent function, in the expression for $X$ (30) we have two independent sums over the internal Matsubara frequencies, that allows one (by decomposition into the simple fractions) to perform the summation over all internal frequencies.

The diagrammatic series (27) and (29) can be expressed in the following analytical forms

$$
\begin{aligned}
& \left\langle S^{z}\right\rangle=\frac{1}{\sqrt{2 \pi X}} \int_{-\infty}^{+\infty} \exp \left(-\frac{\xi^{2}}{2 X}\right) b(y+\xi) d \xi \\
& \Xi=\frac{1}{X \sqrt{2 \pi X}} \int_{-\infty}^{+\infty} \exp \left(-\frac{\xi^{2}}{2 X}\right) \xi b(y+\xi) d \xi
\end{aligned}
$$

As one can see the contribution of diagrammatic series with the 'double-tail' parts corresponds to the average with the Gaussian distribution, where $X$ can be interpreted as a root-mean-squares (r.m.s.) fluctuation of the internal mean field around its mean value $y(14)$. Thus, we obtain a self-consistent set of the equations for the pseudospin mean value (32) and the r.m.s. fluctuation parameter (30) together with the expression for the pseudospin correlation function (31).

The grand canonical potential within the adopted gaussian fluctuation approximation is given by the diagrammatic series

$$
\beta \Omega=\beta \Omega_{\mathrm{GRPA}}
$$

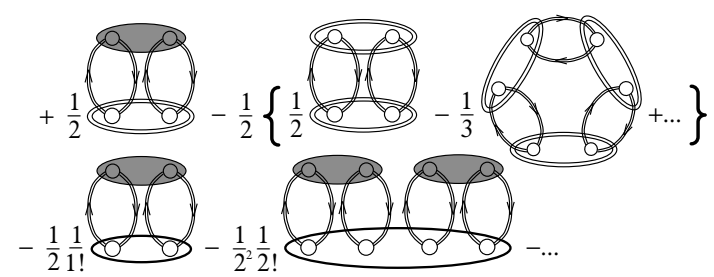

The corresponding analytical expression is

$$
\begin{aligned}
\Omega & =\Omega_{\mathrm{GRPA}} \\
& +\frac{1}{2} \frac{1}{\sqrt{2 \pi X}} \int_{-\infty}^{+\infty} \mathrm{e}^{-\frac{\xi^{2}}{2 X}} \xi b(y+\xi) d \xi
\end{aligned}
$$




$$
\begin{aligned}
& -\frac{1}{2} \int_{-\infty}^{+\infty}\left\{1-\operatorname{erf}\left(\frac{|\xi|}{\sqrt{2 X}}\right)\right\} \operatorname{sign}(\xi) b(y+\xi) d \xi \\
& -\frac{1}{2} \Xi \frac{2}{N} \sum_{n, \boldsymbol{k}} \Lambda_{n}^{2} \tilde{t}_{n}(\boldsymbol{k})^{2} \\
& +\frac{1}{2} \ln \left(1+\Xi \frac{2}{N} \sum_{n, \boldsymbol{k}} \Lambda_{n}^{2} \tilde{t}_{n}(\boldsymbol{k})^{2}\right) .
\end{aligned}
$$

This grand canonical potential satisfies the stationary conditions

$$
\frac{d \Omega}{d X}=0, \quad \frac{d \Omega}{d\left\langle S^{z}\right\rangle}=0
$$

which are equivalent to Eqs. (30),(32). The consistency of the approximation used for the pseudospin mean val- ue, the pseudospin correlation function, and the grand canonical potential can be checked explicitly using the relations

$$
\frac{d \Omega}{d(-h)}=\left\langle S^{z}\right\rangle,\left.\frac{d\left\langle S^{z}\right\rangle}{d(-h \beta)}\right|_{X=\mathrm{const}}=\left\langle S^{z} S^{z}\right\rangle_{\boldsymbol{q}=0}
$$

In the limit of vanishing fluctuations our results go over into the corresponding ones obtained within the selfconsistent GRPA scheme.

The analytical scheme presented in this paragraph (i. e. the gaussian fluctuation approximation), and used for the simplified PEM can be easily reduced to the scheme proposed by the Onyszkiewicz for the pure spin models. For this purpose we consider the renormalization by using the simplest pseudospin correlation function that involves the 'double-tail' fragments of the diagrams, i. e. the first term in Eq. (28)

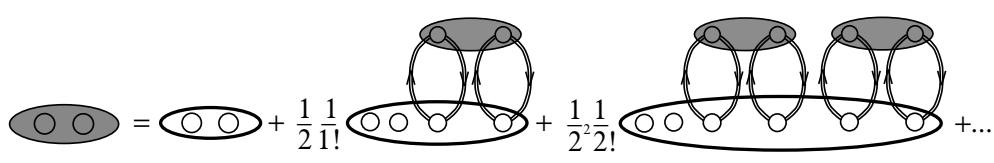

Therefore, in all diagrammatic expressions (27),(29), (30), and (34) we replace the shadowed oval into the new one defined by Eq. (38). Within the framework of this simplification the grand canonical potential satisfies the stationary conditions (36) and can be written in the following analytical form

$$
\begin{aligned}
& \Omega=\Omega_{\mathrm{GRPA}}+\frac{1}{4} \Xi X \\
& -\frac{1}{2} \int_{-\infty}^{+\infty}\left\{1-\operatorname{erf}\left(\frac{|\xi|}{\sqrt{2 X}}\right)\right\} \operatorname{sign}(\xi) b(y+\xi) d \xi .
\end{aligned}
$$

The diagrammatic series for the pseudospin mean value has the same structure as the above presented ones (27), and the final set of equations on the pseudospin mean value and the r.m.s. fluctuation parameter within the Onyszkiewicz type approximation can be written down as

$$
\left\langle S^{z}\right\rangle=\frac{1}{\sqrt{2 \pi X}} \int_{-\infty}^{+\infty} \exp \left(-\frac{\xi^{2}}{2 X}\right) b(y+\xi) d \xi
$$

$$
\begin{aligned}
& \quad X=\left(\frac{2}{N} \sum_{\boldsymbol{k}, n} \Lambda_{n}^{2} \tilde{t}_{n}^{2}(\boldsymbol{k})\right)^{2} \\
& \quad \times \frac{1}{X \sqrt{2 \pi X}} \int_{-\infty}^{+\infty} \exp \left(-\frac{\xi^{2}}{2 X}\right) \xi b(y+\xi) d \xi \text {. } \\
& \text { IV. PHASE DIAGRAMS WITHIN THE } \\
& \text { GAUSSIAN FLUCTUATION APPROACH } \\
& \text { In this paragraph we present the results of numerical } \\
& \text { solution of equations for the pseudospin mean value and } \\
& \text { the r.m.s. fluctuation parameter within all the above de- } \\
& \text { scribed methods (the self-consistent GRPA, the gaussian } \\
& \text { fluctuation approximation, and the Onyszkiewicz type } \\
& \text { approximation). We consider the regime of fixed chemical } \\
& \text { potential value ( } \mu \text { = const), and deal with the simplified } \\
& \text { PEM on a square lattice with the nearest-neighbour hop- } \\
& \text { ping. The stable state of the system is described by the } \\
& \text { solution that corresponds to the global minimum of the } \\
& \text { grand canonical potential; metastable states are related } \\
& \text { to the solutions that correspond to the local minima. }
\end{aligned}
$$


a)

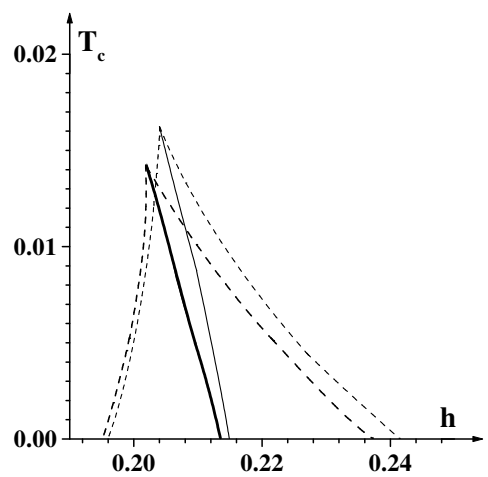

b)

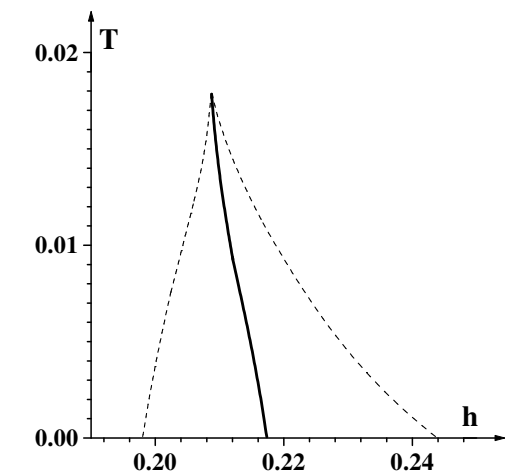

Fig. 3. $T_{\mathrm{c}}-h$ phase diagrams within the a) gaussian fluctuation methods, b) self-consistent GRPA scheme $(g=1$, $\left.t_{k=0}=0.2, \mu=-0.4\right)$.

We note that there are no particular differences between the results obtained within the framework of the self-consistent gaussian fluctuation approximation (by solving the set of Eqs. (30),(32)) and the Onyszkiewicz type approximation (by solving the set of Eqs. (39),(40)). The representative results are shown on the first order phase diagram (figure 3a) where thick solid line is the result of the calculations within the Onyszkiewicz type approximation, and thin solid line is the result of the gaussian fluctuation approximation. We can see only a minor quantitative difference between these two phase transition lines.

In general, for a wide range of the model parameters we have found that both 'full' the self-consistent gaussian fluctuation approximation and its 'simplified version' the Onyszkiewicz type approximation give the same topological type and slope of phase diagrams, a similar field and temperature behaviours of the pseudospin mean value and the grand canonical potential. Therefore, to show the influence of the fluctuation effects on the thermodynamic properties of the simplified PEM we present all the results within the Onyszkiewicz type approximation. With this latter approximation it is simple to complete numerical calculations, this approximation leads to the lowest value of temperature in the critical point, and represents all features of the self-consistent gaussian fluctuation approximation. a)

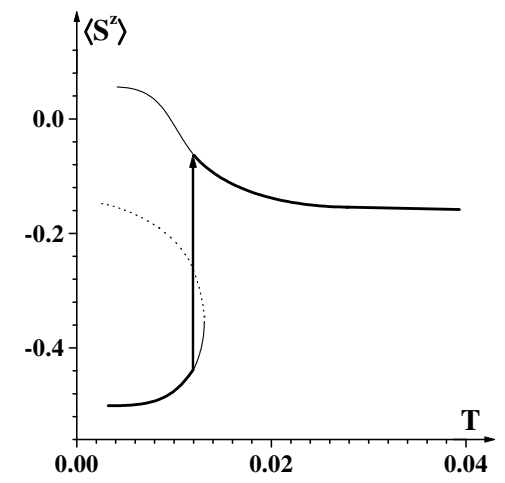

b)

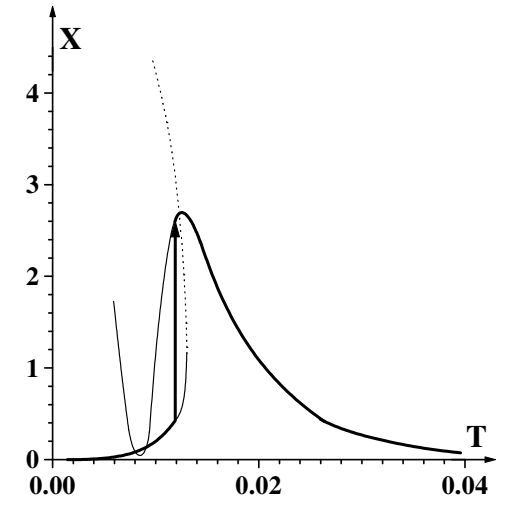

Fig. 4. Temperature dependences of (a) pseudospin mean value and (b) r.m.s. fluctuation parameter $\left(g=1, t_{\boldsymbol{k}=0}=0.2\right.$, $\mu=-0.4, h=0.204)$. Thick solid lines correspond to thermodynamically stable states, other lines denote metastable and unstable ones.

In figures $3 \mathrm{a}$ and $3 \mathrm{~b}$ we present the first order phase transition diagrams within the different approximations in the case when the chemical potential is fixed and placed within the lower electron subband $\mu=-0.4$. As one can see, taking the fluctuations into account (figure 3a) does not change qualitatively the results obtained within the self-consistent GRPA scheme (figure 3b). Such a situation is typical in the case when the chemical potential is placed within the lower/upper subband of the electron spectrum except for some special cases described below. The quantitative changes due to fluctuations are important in the region of the critical point, fluctuations lead to a significant lowering of the critical point temperature: $T=0.018$ for the self-consistent GRPA method (in figure $3 \mathrm{~b}$ ), and $T=0.0145$ for the Onyszkiewicz type approximation (in figure $3 \mathrm{a}$ ).

Typical temperature behaviours of the pseudospin mean value and the r.m.s. fluctuation parameter within the Onyszkiewicz type approximation are presented in figures $4 \mathrm{a}, \mathrm{b}$ for the fixed value of the field $h=0.204$ and for the chemical potential $\mu=-0.4$ (same as in figures 3). At increasing temperature the pseudospin mean value jumps from the branch of low temperature phase to the branch of high temperature phase in the phase transition point $T=0.0109$, and the r.m.s. fluctuation parameter rapidly changes its magnitude by about seven times. 
A certain qualitative change takes place when the chemical potential is placed near the center of the electron subband $(\mu=-g / 2=-0.5)$.

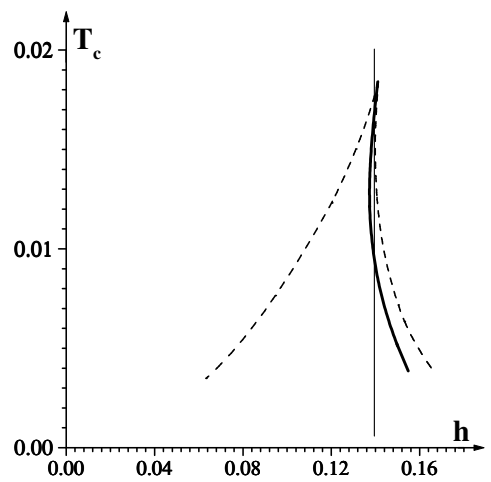

Fig. 5. $T_{\mathrm{c}}-h$ phase diagrams within the Onyszkiewicz type approximation $\left(g=1, t_{k=0}=0.2, \mu=-0.5\right)$.

Comparing with the self-consistent GRPA or the DMFA (figure 2a,b) a change of slope of the phase transition curve is observed (figure 5). The vertical line on the $T_{\mathrm{c}}-h$ phase diagram twice crosses the phase transition curve (thick solid line in figure 5), and, hence, there exists a possibility of two sequential first order phase transitions with the change of temperature (reentrance).
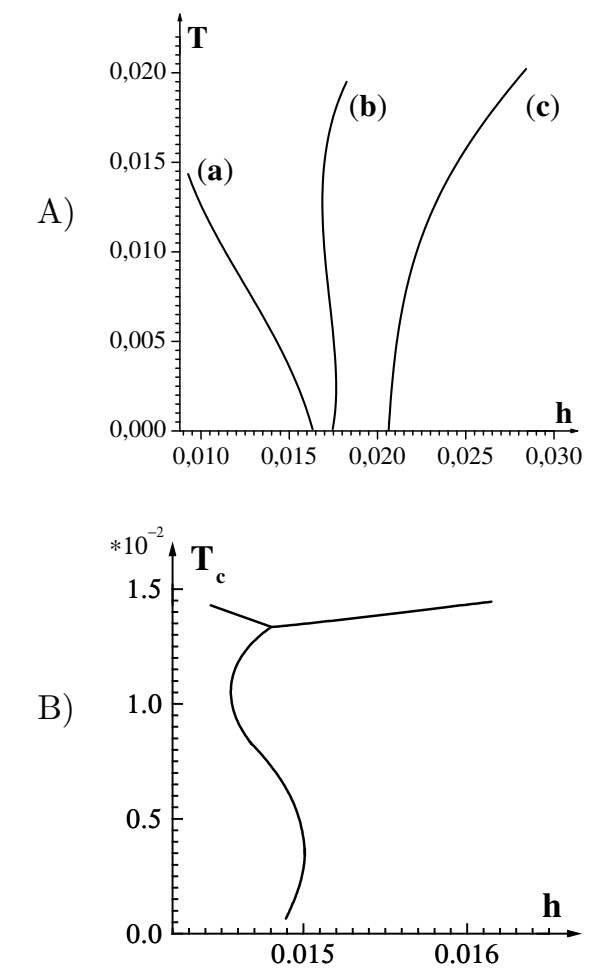

Fig. 6. $T_{\mathrm{c}}-h$ phase diagrams within the A) self-consistent GRPA: (a) $\mu=-0.588$, (b) $\mu=-0.6$, (c) $\mu=-0.613$; B) the Onyszkiewicz type approximation $\left(g=1, t_{k=0}=0.2\right.$, $\mu=-0.6)$. a)

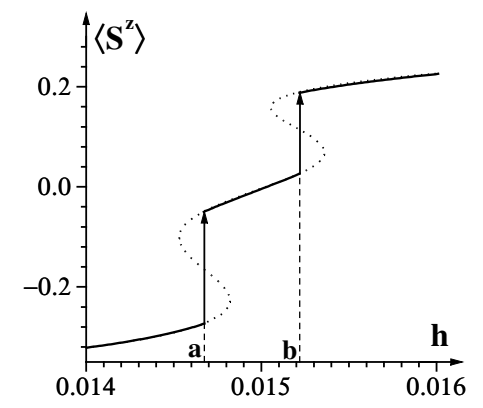

b)

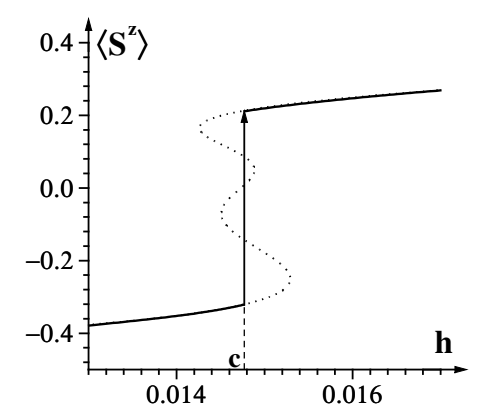

Fig. 7. Field dependences of the pseudospin mean value a) above the triple point $(T=0.01370)$, b) below the triple point $(T=0.01316)\left(g=1, t_{k=0}=0.2, \mu=-0.6\right)$. 
a)

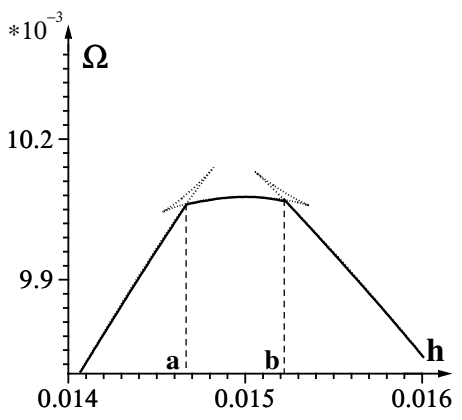

b)

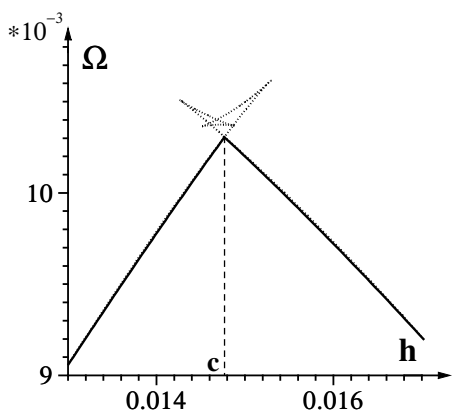

Fig. 9. Field dependences of the grand canonical potential a) above the triple point $(T=0.01370)$, b) below the triple point $(T=0.01316)\left(g=1, t_{k=0}=0.2, \mu=-0.6\right)$.

a)

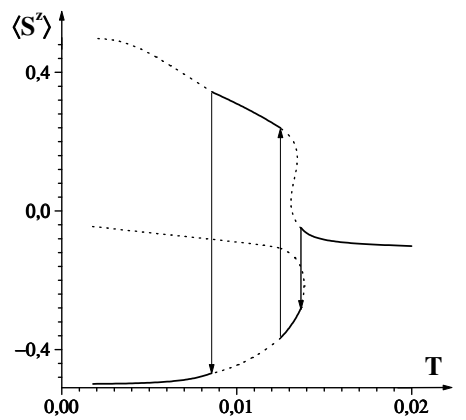

b)

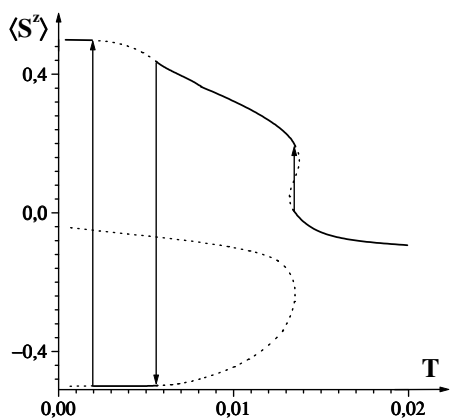

Fig. 10. Temperature dependences of the pseudospin mean value a) $h=0.01467$, b) $\quad h=0.01495\left(g=1, t_{k=0}=0.2\right.$, $\mu=-0.6)$.

Further, we find that for the different values of the chemical potential a slope of the phase transition curve can vary: see figure $6 \mathrm{~A}$ where we present phase tran- sition curves for three deferent chemical potential values in the self-consistent GRPA calculations. Within the region, where the change of phase curve slope occurs $(\mu \simeq-0.6 \pm 0.005)$ a possibility of three sequential phase transitions of the first order at change of temperature is observed (phase diagram in figure $6 \mathrm{~A}(\mathrm{~b})$ ). In this region the qualitative changes, i.e. the change of the topological type of the first order phase transition curve and the appearance of the triple point are observed when we include the gaussian fluctuations in calculations (see figure $6 \mathrm{~B}$ ). Therefore, the fluctuation effects become significant when the chemical potential has a value within the mentioned range. This serves to note that the described situation occurs also in the case when the chemical potential is placed within the other region of the lower electron subband $\mu \simeq-0.425 \pm 0.005$.

The field dependences of the pseudospin mean value and the r.m.s. fluctuation parameter in the temperature vicinity of the triple point are presented in figures 7 and 8 , both above and below the triple point. The phase transition points (denoted as $\mathbf{a}, \mathbf{b}$ and $\mathbf{c}$ points in figures 7,8$)$ correspond to the crossing points of different branches of the grand canonical potential $\Omega(h)$ in figure 9 . Figures $7 \mathrm{a}-9 \mathrm{a}$ demonstrate the situation the with temperature above the triple point. We find two sequential phase transitions: at the point $\mathbf{a}$ the transition to the intermediate phase with the pseudospin mean value $S^{z} \sim 0$ and large fluctuations occurs; then at increasing field one has the transition to the phase with $S^{z} \simeq 0.2$ and small fluctuations. The existence of the stable intermediate solution can be interpreted as a manifestation of instability with respect to phase modulation. Below the triple point the intermediate state is metastable, and we see one phase transition (figures $7 \mathrm{~b}-9 \mathrm{~b}$ ). In figures 10a,b we present the temperature dependences of the pseudospin mean value at the fixed field from the left and right of the triple point. In both cases three sequential first order phase transitions are found.

Let us recall that in all above - presented figures we considered the case when chemical potential is placed within the lower electron subband. We established that the self-consistent gaussian fluctuation approximation and the Onyszkiewicz type approximation do not violate the electron-hole symmetry in the simplified PEM. Therefore, if the chemical potential is placed in the upper subband our results transform according to the mentioned above electron-hole symmetry (25) of the initial Hamiltonian.

\section{SUMMARY AND DISCUSSION}

To summarize, we have presented a theoretical investigation of the simplified PEM in the strong coupling limit $(g \gg t)$. The self-consistent gaussian fluctuation approximation, i. e. the approximation that takes into account the corrections of the gaussian fluctuations to the selfconsistent GRPA, has been employed to study the first order phase transitions in the simplified PEM. This approximation allows one to calculate the grand canonical 


\section{K. V. TABUNSHCHYK}

potential, the pseudospin mean value together with the pseudospin correlation function. We have also reduced the self-consistent gaussian fluctuation approximation to the Onyszkiewicz type approximation by taking into account the restricted class of diagrams on the analogy with the Onyszkiewicz's works [26,27]. The Onyszkiewicz approximation was originally proposed for the spin models, and is more suitable to perform numerical calculations. Moreover, the Onyszkiewicz approximation yields a much better description of critical properties of the spin models in comparison with other approaches.

We have analyzed the grand canonical potential, the behaviour of pseudospin mean value and r.m.s. fluctuation parameter within both full gaussian fluctuation approximation and restricted the Onyszkiewicz type approximation in a wide range of temperature and model parameters. Our results have demonstrated that the Onyszkiewicz approximation does not change qualitatively any of the results obtained within the full gaussian fluctuation approximation. Further, we find that the lowest temperature in the critical points corresponds to the Onyszkiewicz type approximation.

By comparing the results of calculations in the mean field type approximations (self-consistent GRPA, DMFA) with the Onyszkiewicz approximation we conclude that the fluctuation effects are very strong near the critical point, and lead to the quantitative and for certain chemical potential values to the qualitative changes in the behavior of thermodynamical functions and in the shape of phase diagrams. We have obtained that fluctuations lower the critical point temperature and shift the corresponding value of the longitudinal field. We have found that the triple point can appear on the phase transition curve due to the fluctuations, and the transition to the phase with pseudospin mean value $S^{z} \sim 0$ and very high fluctuations occurs in the vicinity of the triple point.
Our preliminary analysis of temperature behaviour of the pseudospin correlation function $\left\langle S^{z} S^{z}\right\rangle_{\boldsymbol{q}}$ (31) (with the fixed r.m.s. fluctuation parameter) shows that the high temperature phase become unstable with respect to the fluctuations with the wave vector $\boldsymbol{q} \neq 0$ in the case when chemical potential $\mu$ is placed between the electron subbands, i. e. in the gap of the spectrum. The maximal temperature of instability is achieved for $\boldsymbol{q}=(\pi, \pi)$, that indicates a possibility of phase transition into a modulated chessboard phase. This result confirms the previously obtained one within the framework of the self-consistent GRPA [17, 18], but taking the fluctuations into account noticeably lowers the temperature where the instability occurs. Therefore, we conclude that fluctuations lead to the narrowing of the temperature range where modulated phase can be found.

In this paper we investigated possible phase transitions in PEM within the gaussian fluctuation approximation without the creation of super structures, and all the presented phase diagrams concern only this case $(\boldsymbol{q}=0)$. However, presented in the paper the gaussian fluctuations method as well as the Onyszkiewicz approximation can be used to investigate the influence of fluctuations on the thermodynamic properties of modulated chessboard phase just as it was done in [18] within the self-consistent GRPA. This issue will be the subjected of a further investigation.

\section{ACKNOWLEDGEMENT}

The author wishes to thank Professor I. V. Stasyuk and Dr. O. V. Velychko for their helpful discussions and for a critical reading of the paper. This work was partially supported by the Fundamental Research Fund of the Ministry of Ukraine for Science and Education (Project No. $02.07 / 266)$.
[1] J. Mustre de Leon, S. D. Conradson, I. Batistic, A. R. Bishop, I. D. Raistrick, M. C. Aronson, F. H. Garzon, Phys. Rev. B 45, 2447 (1992).

[2] N. Poulakis, D. Palles, E. Liarokapis, K. Conder, E. Kaldis, K. A. Müller Phys. Rev. B 53, R534 (1996).

[3] M. N. Iliev, V. G. Hadjiev, V. G. Ivanov, J. Raman Spectr. 27, 333 (1996).

[4] R. J. Cava, A. W. Hewat, E. A. Hewat, B. Batlogg, M. Marezio, K. M. Rabe, J. J. Krajewski, W. F. Peck, Jr., L. W. Rupp, Jr., Physica C: Superconductivity 165, 419 (1990).

[5] K. Müller, Z. Phys. B 80, 193 (1990).

[6] J. Hirsch, Phys. Lett. A 134, 451 (1989).

[7] J. Hirsch, S. Tang, Phys. Rev. B 40, 2179 (1989).

[8] E. Matsushita, Phys. Rev. B 51, 17332 (1995).

[9] E. Matsushita, Ferroelectrics 203, 349 (1997).

[10] M. Frick, W. von der Linden, I. Morgenstern, H. Raedt, Z. Phys. B 81, 327 (1990).

[11] I. V. Stasyuk, A.M. Shvaika, Schachinger, Physica C 213, 57 (1993).
[12] I. V. Stasyuk, A. M. Shvaika, Condens. Matter Phys. 3, 134 (1994).

[13] I. V. Stasyuk, A. M. Shvaika, O. Danyliv, Mol. Phys. Rep. 9, 61 (1995).

[14] I. V. Stasyuk, A. M. Shvaika, Czech. J. Phys. 46, 961 (1996).

[15] I. V. Stasyuk, A. M. Shvaika, J. Phys. Stud. 3, 177 (1999); preprint arXiv: cond-mat/9807353.

[16] I. V. Stasyuk, A. M. Shvaika, K. V. Tabunshchyk, Condens. Matter Phys. 2, 109 (1999); preprint arXiv: condmat/0006100.

[17] I. V. Stasyuk, A. M. Shvaika, K. V. Tabunshchyk, Acta Phys. Pol. A 97, 411 (2000).

[18] I. V. Stasyuk, A. M. Shvaika, K. V. Tabunshchyk, Ukr. Phys. J. 45, 520 (2000); preprint arXiv: condmat/0010334.

[19] I. V. Stasyuk, O. Danyliv, Phys. Status Solidi B 299, 299 (2000); preprint arXiv: cond-mat/0204373.

[20] J. K. Freericks, R. Lemanski, Phys. Rev. B 61, 13438 (2000). 
[21] J. K. Freericks, Phys. Rev. B 47, 9263 (1993).

[22] B. M. Letfulov, Eur. Phys. J. B 11, 423 (1999).

[23] J. K. Freericks, Ch. Gruber, N. Macris, Phys. Rev. B 60, 1617 (1999); preprint arXiv: cond-mat/9901289

[24] U. Brandt, C. Mielsch, Z. Phys. B - Cond. Matter, 75, 365 (1989); 79, 295 (1990); 82, 37 (1991).

[25] I. V. Stasyuk, K. V. Tabunshchyk, Condens. Matter. Phys. 4, 109 (2001).

[26] Z. Onyszkiewicz, Phys. Lett. 57A, 480 (1976).
[27] Z. Onyszkiewicz, Phys. Lett. 76A, 411 (1980).

[28] Yu. Izyumov, B. Letfulov, J. Phys.: Condens. Matter 2, 8905 (1990).

[29] P. Slobodyan, I. Stasyuk, Teor. Mat. Fiz. 19, 423 (1974). [Theor. Math. Phys. 19, 616 (1974)].

[30] Yu. Izyumov, F. Kassan-Ogly, Yu. Skriabin, J. de Phys. 325, 1 (1971).

[31] Yu. Izyumov, F. Kassan-Ogly, Yu. Skriabin, Fields Methods in the Theory of Ferromagnets (Mir, Moscow, 1974).

\title{
ТЕРМОДИНАМІЧНІ ВЛАСТИВОСТІ ПСЕВДОСПІН-ЕЛЕКТРОННОЇ МОДЕЛІ В НАБЛИЖЕНІ ГАУССОВИХ ФЛЮКТУАЦІЙ
}

\author{
K. В. Табунщик \\ Інститут фізики конденсованих систем Національної академї наук Украӥни, \\ вул. Свєнціцького, 1, Лъвів, 79011, Украӥна
}

Досліджено вплив ефектів, пов'язаних із флюктуаціями внутрішнього ефективного поля на термодинаміку фазових переходів у псевдоспін-електронній моделі. У межах самоузгодженого методу врахування ґауссових флюктуацій (на основі схеми узагальненого наближення хаотичних фаз (УНХФ)), а також у наближенні типу Онишкевича розраховано термодинамічний потенціял, середнє значення оператора псевдоспіну та величину середньоквадратичної флюктуації внутрішнього ефективного поля.

Розрахунок термодинамічних функцій показав, що, на відміну від спінових моделей з прямою взаємодією (коли повне врахування гауссових флюктуацій приводить до появи фіктивних фазових переходів, а в наближенні Онишкевича вони відсутні), у псевдоспін-електронній моделі, де взаємодія має непрямий багаточастинковий характер, наближення типу Онишкевича не змінює якісно результатів, отриманих у межах самоузгодженого наближення гауссових флюктуацій.

Порівняння з наближенням УНХФ продемонструвало, що врахування флюктуацій приводить до певних змін у розташуванні кривої рівноваги фаз і є найбільш суттєвим в околі критичної точки (відбувається пониження температури критичної точки і зсув відповідного значення поздовжнього поля; найнижче значення критичної температури одержано в наближенні типу Онишкевича). При певних значеннях хемічного потенціялу (в ділянці зміни нахилу кривої рівноваги фаз) флюктуації можуть приводити до якісних змін у поведінці термодинамічних функцій. Так, зокрема, на польовій залежності найнижчої гілки термодинамічного потенціялу виникають дві точки зламу, у яких існує стрибок параметра порядку. Така поведінка відображається у зміні топології фазової діяграми, що проявляється, зокрема, у появі двох критичних точок. 